\title{
Influence of speckle image reconstruction on photometric precision for large solar telescopes
}

\author{
C. L. Peck ${ }^{1,2}$, F. Wöger ${ }^{3}$, and J. Marino ${ }^{3}$ \\ ${ }^{1}$ Laboratory for Atmospheric and Space Physics, Boulder CO, 80303, USA \\ e-mail: courtney.peck@colorado.edu \\ 2 Department of Physics, University of Colorado, Boulder CO, 80303, USA \\ 3 National Solar Observatory, Boulder CO, 80303, USA
}

Received 23 May 2017 / Accepted 6 September 2017

\begin{abstract}
Context. High-resolution observations from large solar telescopes require adaptive optics (AO) systems to overcome image degradation caused by Earth's turbulent atmosphere. AO corrections are, however, only partial. Achieving near-diffraction limited resolution over a large field of view typically requires post-facto image reconstruction techniques to reconstruct the source image.

Aims. This study aims to examine the expected photometric precision of amplitude reconstructed solar images calibrated using models for the on-axis speckle transfer functions and input parameters derived from AO control data. We perform a sensitivity analysis of the photometric precision under variations in the model input parameters for high-resolution solar images consistent with four-meter class solar telescopes.

Methods. Using simulations of both atmospheric turbulence and partial compensation by an AO system, we computed the speckle transfer function under variations in the input parameters. We then convolved high-resolution numerical simulations of the solar photosphere with the simulated atmospheric transfer function, and subsequently deconvolved them with the model speckle transfer function to obtain a reconstructed image. To compute the resulting photometric precision, we compared the intensity of the original image with the reconstructed image.

Results. The analysis demonstrates that high photometric precision can be obtained for speckle amplitude reconstruction using speckle transfer function models combined with AO-derived input parameters. Additionally, it shows that the reconstruction is most sensitive to the input parameter that characterizes the atmospheric distortion, and sub-2\% photometric precision is readily obtained when it is well estimated.
\end{abstract}

Key words. techniques: image processing - techniques: interferometric - Sun: photosphere

\section{Introduction}

The next generation of solar telescopes, including the currently under-construction $4 \mathrm{~m}$ Daniel K. Inouye Solar Telescope (DKIST; Elmore et al. 2014; Tritschler et al. 2016) and the upcoming $4 \mathrm{~m}$ European Solar Telescope (EST; Collados et al. 2013; Matthews et al. 2016), will observe the solar surface with unprecedented $\sim 15 \mathrm{~km}$ resolution in the visible spectrum, constituting a near threefold improvement over current solar telescopes. Achieving diffraction-limited resolution requires correction of seeing degradation due to Earth's turbulent atmosphere using an $\mathrm{AO}$ system. $\mathrm{AO}$ correction, however, remains only partial due to the limited bandwidth of the AO system's control loop and the finite number of sensing and correcting elements in the system. Furthermore, outside the isoplanatic path, the wavefront remains largely uncorrected because of the spatial decorrelation of atmospheric turbulence at different viewing angles. Multi-conjugate adaptive optics (MCAO) offer the potential to overcome the latter issue (e.g., von der Lühe et al. 2005; Berkefeld et al. 2006; Schmidt et al. 2017), but residual errors will always be present to some degree. Reaching the diffraction limit both inside and outside the isoplanatic patch, therefore requires post-facto image reconstruction techniques such as multiobject multi-frame blind deconvolution (e.g., van Noort et al. 2005) and speckle interferometry, the latter of which is the focus of this work.
Speckle image reconstruction utilizes a sequence of short exposure images - a speckle burst - to reconstruct the unperturbed source image. Since the atmosphere typically evolves on much smaller timescales than solar structures $(\sim 10 \mathrm{~ms}$ and $100 \mathrm{~s}$, respectively), image exposures on the order of atmospheric evolution timescales capture the turbulent motion of the atmosphere over the stationary source. Each burst image contains some information on small-scale atmospheric structures, which is severely attenuated for long-exposure images. Using a collection of short-exposure burst images, the smallscale structure of the source can be recovered by separately reconstructing the source's Fourier phases and amplitudes. The Fourier phases are estimated using the Knox-Thompson crossspectrum (Knox \& Thompson 1974) or triple-correlation bispectra (Lohmann et al. 1983), which show good convergence for the solar case (de Boer et al. 1992; Wöger et al. 2008). The Fourier amplitudes are reconstructed using Labeyrie's method (Labeyrie 1970), and are the focus of this work.

Fourier amplitude reconstruction requires knowledge of the speckle transfer function (STF), which includes individual contributions from the telescope, $\mathrm{AO}$ system, and atmosphere over the speckle burst. Generally, the telescope can be wellcharacterized, but the atmospheric and $\mathrm{AO}$ system contributions to the STF must be estimated or measured directly. Since solar observations cannot make use of calibration stars as done in night-time astronomy, the atmospheric contribution to the STF is 
estimated by modeling the index of refraction fluctuations due to turbulence and their effects on image quality. These turbulence effects can be characterized by a single parameter known as the Fried parameter (Fried 1966), $r_{0}$, which quantifies the strength of the atmospheric turbulence. Given an estimate of $r_{0}$, the atmospheric STF can be solved analytically (Korff 1973). For observations using an AO system, the atmospheric STF is modified to include the partial wavefront compensation through AO performance characteristics that estimate the wavefront correction (Wang \& Markey 1978; Wöger \& von der Lühe 2007).

To estimate $r_{0}$, von der Lühe (1984) proposed the spectral ratio method, which uses the ratio of the equivalent long-exposure power spectrum to the average short-exposure power spectrum of the images that constitute the speckle burst. This method is currently used in speckle reconstruction algorithms at several ground-based solar observatories with good success (e.g., Mikurda \& von der Lühe 2006; Denker et al. 2007; Wöger et al. 2008) and with modifications to include a fixed estimate for partial compensation by an AO system and to account for anisoplanatism (Puschmann \& Sailer 2006; Wöger \& von der Lühe 2007). However, a systematic study of the influence of the STF input parameters ( $r_{0}$ and $\mathrm{AO}$ performance parameters) on the photometric precision of the reconstructed image is still lacking. Such a study is of particular relevance in light of results from Denker et al. (2007) showing that the estimation of $r_{0}$ is impacted by the morphological structure of the scenery observed through azimuthal asymmetry in the image power spectrum and is potentially inaccurate. This image-scene dependence will likely have a greater impact on upcoming high-resolution observations, since the ratio of $r_{0}$ to the telescope aperture is smaller and therefore requires a more accurate measurement of $r_{0}$.

This paper presents a speckle reconstruction model that estimates $r_{0}$ from the AO system control data, which removes the potential of image scene bias in the estimation of $r_{0}$. We performed an in-depth study of the sensitivity of the modeled onaxis STF to the $r_{0}$ estimate and AO performance parameters applicable for four-meter class solar telescopes. Using simulations of atmospheric turbulence of a known $r_{0}$ and a simulated $\mathrm{AO}$ system, we computed $r_{0}$ and the AO performance parameters using AO system measurements. We then separately varied the input parameters and computed the resulting model STFs. We deduced the sensitivity of the photometric precision to the input parameters by convolving high-resolution solar surface images with the simulated atmosphere-telescope STF and subsequently deconvolving with a model STF and comparing the results with the original image. Section 2 describes the details of the simulations used in this study. Section 3 details the methods used to construct and test the transfer functions. Section 4 describes the results of the sensitivity analysis. Finally, Sect. 5 discusses the results in the context of future high-resolution observations including extension to MCAO systems.

\section{Simulations and analytical models}

In this section, we describe the atmospheric and AO system simulations, as well as the analytical STF model. The atmospheric simulation implements the Kolmogorov power spectrum to simulate wavefront distortions through Earth's atmosphere. The simulated AO system subsequently measures these wavefront distortions with a wavefront sensor (WFS) and creates a post-AO compensated, or residual, wavefront using a model for the deformable mirror (DM). The WFS measurements and DM actuator commands are used to compute $r_{0}$ and the AO performance parameters, which are then input to the model STF.

\subsection{Atmospheric simulation}

The AO simulations rely on an atmospheric model to reproduce the effects of atmospheric turbulence. The atmospheric model used in our simulations was a simple model composed of a single turbulent layer located at the telescope entrance pupil, which was scaled for Fried parameters of $r_{0}=10 \mathrm{~cm}$ and $15 \mathrm{~cm}$. The layer is represented by a large phase screen of $8192 \times 8192$ pixels that slides across the telescope aperture from right to left to simulate the effect of wind with a constant speed of $5 \mathrm{~m} \mathrm{~s}^{-1}$. For simplicity, we assumed a constant wind speed throughout the full simulation. The phase screen was computed using a FFT-based method (Johansson \& Gavel 1994), which combines a known power spectrum of the phase (Kolmogorov power spectrum) with a random Fourier phase.

This computation method is known to under-represent low spatial frequencies, corresponding to low order Zernike or Karhunen-Loève (KL) modes (for the definition of Zernike or KL modes see e.g., Wang \& Markey 1978; Dai 1995). This effect can be compensated by the addition of low spatial frequency sub-harmonics to the phase. In our case, it was determined that this correction to the phase screen was not necessary because of the small size of the pupil $(512 \times 512$ pixels $)$ in comparison to the full size of the phase screen. By not adding sub-harmonics to the phase screen we ensured that it remained cyclically continuous and can be tiled to produce an effectively infinite phase screen. These infinite phase screens, where the phase wraps around at the edges indefinitely, can be used to produce very long time series of continuous $\mathrm{AO}$ simulations.

\subsection{Adaptive optics simulation}

We used a solar AO simulation tool developed at the National Solar Observatory (Marino 2012) to simulate the operation of the AO system currently under development for the DKIST. The AO simulation package provides a flexible environment to simulate any configuration of classical or multi-conjugate AO systems. It allows an arbitrary number of WFS and active mirrors that can accommodate almost any system configuration. The AO simulation package fully simulates field dependent effects like anisoplanatism. Although, it should be noted that for this study we only considered the on-axis performance of the simulated AO system. The results of this study are then only valid inside the isoplanatic patch, which is typically of the order of 5 to $10^{\prime \prime}$ (Denker et al. 2007).

The simulated AO system for this work replicates the design of the actual DKIST AO system. It consists of a WFS and two active mirrors: a dedicated tip-tilt mirror and a DM. The simulated WFS is a cross-correlating Shack Hartmann (SH) WFS with 1429 sub-apertures placed in a $43 \times 43$ square grid. The simulated DM is a continuous face plate mirror with a total of 1516 actuators located in a $44 \times 44$ square grid. The DKIST DM is equipped with 1600 actuators, some of which fall outside the DM aperture and are slaved to their nearest neighbors (see e.g., Johnson et al. 2014, and references therein). For simplicity, we ignored these slaved actuators and only included actively controlled actuators. The WFS and DM are placed in the Fried geometry where the actuators of the DM are aligned with the corners of the WFS sub-apertures. Due to the computationally intensive nature of the problem, the code that simulates the solar cross-correlating SH WFS does not actually perform the cross-correlations between the WFS sub-aperture images. Instead, the AO simulation tool computed the average tip and tilt from the phase screen portion in each WFS subaperture to 
calculate the shift value for that subaperture. Numerical tests validated that this algorithmic simplification produces very similar results to a typical extended source SH WFS.

The simulated AO system must be calibrated before it can be operated. Calibration was performed by measuring the response of the WFS to a set of known inputs placed on the active mirrors, as is normally done in real AO systems. In our simulations, we used KL modes to perform the calibration and built a least-squares wavefront reconstructor using the Moore-Penrose pseudo-inverse (Gavel 2003). The AO control matrix obtained by this process was used to reconstruct the wavefront measured by the WFS. The wavefront was reconstructed as a linear combination of $500 \mathrm{KL}$ modes, which are projected onto the DM actuators. A simulated servo loop integrated the reconstructed residual wavefront at each iteration to produce an optimal update to the shape of the mirrors.

The simulated AO system operates nominally at a frequency of $2 \mathrm{kHz}$. We ran our simulated $\mathrm{AO}$ system for a total of 10000 iterations, corresponding to a total simulated time of $5 \mathrm{~s}$. At each iteration we recorded the distorted uncorrected wavefront phase produced by the atmosphere model and the residual phase after correction by the AO system. The uncorrected atmospheric phases provide information about the exact seeing conditions the AO system was attempting to correct, while the residual phases provide information about the quality of the correction supplied by the simulated AO system. The data from the residual phase facilitate the direct computation of the STF and therefore set the "ground truth" for a test of the analytical STF models and their input.

\subsection{Analytical model for Fourier amplitude reconstruction}

The STF is used in post-facto image reconstruction techniques that require the calibration of the spatial Fourier amplitudes as a step in the reconstruction process. In particular, the Fourier amplitude recovery in a solar speckle image reconstruction is performed using Labeyrie's method (Labeyrie 1970) applied to post-AO corrected burst images $I_{j}(s)$ where

$$
\left\langle\left|I_{j}(s)\right|^{2}\right\rangle=|O(s)|^{2} \times\left\langle\left|S_{j}(s)\right|^{2}\right\rangle .
$$

The spatial power spectrum of the object $|O(s)|$ can therefore be determined if the STF $\left\langle\left|S_{j}(s)\right|^{2}\right\rangle$ is known. Using the definition of the optical transfer function $S(s)$ and the abbreviation

$g^{\left[{ }^{\prime}\right]+}:=g\left(\boldsymbol{r}^{[\prime]}+\frac{1}{2} s\right) \quad$ and $\quad g^{[\prime]-}:=g\left(\boldsymbol{r}^{[\prime]}-\frac{1}{2} s\right)$

where $g$ is an arbitrary function, the STF is defined by taking the time average over the residual phase after AO correction, as follows:

$$
\begin{aligned}
\left\langle|S(s)|^{2}\right\rangle= & \iint \mathrm{d} \boldsymbol{r}^{\prime} \mathrm{d} \boldsymbol{r} W^{+} W^{-} W^{\prime+} W^{\prime-} \\
& \times\left\langle\operatorname { e x p } \left[-i\left\{\left(\varphi^{+}-\phi^{+}\right)-\left(\varphi^{-}-\phi^{-}\right)\right.\right.\right. \\
& \left.\left.\left.-\left(\varphi^{\prime+}-\phi^{++}\right)-\left(\varphi^{\prime-}+\phi^{\prime-}\right)\right\}\right]\right\rangle,
\end{aligned}
$$

The phase terms can be represented in terms of basis functions, $M_{i}(\boldsymbol{r})$, that are mutually orthogonal on the (non-complex) aperture function $W(\boldsymbol{r})$ as

$\varphi(\boldsymbol{r})=\sum_{i=2}^{\infty} a_{i} M_{i}(\boldsymbol{r}) \quad$ and $\quad \phi(\boldsymbol{r})=\sum_{i=2}^{N} \beta_{i} a_{i} M_{i}(\boldsymbol{r})$.
Here $\varphi(\boldsymbol{r})$ is the phase disturbance of the atmosphere and $\phi(\boldsymbol{r})$ is the AO partial compensation to that phase disturbance. The term $a_{\mathrm{i}}$ represents the expansion coefficient of the $i$ th mode $M_{i}$, and $\beta_{i}$ describes the degree of compensation by the AO system, typically with $-1<\beta_{i}<1$. For example, $\beta_{i}=0$ represents a mode that is entirely uncorrected, while $\beta_{i}=1$ represents a mode that is fully corrected by the AO system. A negative value for $\beta_{i}$ can occur for modes that are not well sampled by the WFS and in anisoplanatic conditions for which the atmospheric modes decorrelate with increasing off-axis viewing angles.

Following the notation of Wöger et al. (2008) and using KL modes - that by definition have a diagonal covariance matrix for the atmospheric expansion coefficients $a_{i}$-as the $M_{i}$, we obtain as the model STF:

$$
\begin{aligned}
\left\langle|S(s)|^{2}\right\rangle= & \iint \mathrm{d} \boldsymbol{r}^{\prime} \mathrm{d} \boldsymbol{r} W^{+} W^{-} W^{\prime+} W^{\prime-} \\
& \times \exp \left[-\mathscr{D}(\boldsymbol{s})-\mathscr{D}(\Delta \boldsymbol{r})+\frac{1}{2}\{\mathscr{D}(\Delta \boldsymbol{r}+\boldsymbol{s})+\mathscr{D}(\Delta \boldsymbol{r}-\boldsymbol{s})\}\right. \\
& \left.+K(\boldsymbol{r}, \boldsymbol{s})+K\left(\boldsymbol{r}^{\prime}, \boldsymbol{s}\right)-\tilde{K}\left(\boldsymbol{r}, \boldsymbol{r}^{\prime}, \boldsymbol{s}\right)-\tilde{K}\left(\boldsymbol{r}^{\prime}, \boldsymbol{r}, \boldsymbol{s}\right)\right]
\end{aligned}
$$

where

$$
\begin{aligned}
& K(\boldsymbol{r}, \boldsymbol{s})=\sum_{i=2}^{N} \beta_{i}\left(1-\frac{1}{2} \beta_{i}\right)\left\langle a_{i}^{2}\right\rangle\left(M_{i}^{+}-M_{i}^{-}\right)\left(M_{i}^{+}-M_{i}^{-}\right) \\
& \tilde{K}\left(\boldsymbol{r}, \boldsymbol{r}^{\prime}, \boldsymbol{s}\right)=\sum_{i=2}^{N} \beta_{i}\left(1-\frac{1}{2} \beta_{i}\right)\left\langle a_{i}^{2}\right\rangle\left(M_{i}^{+}-M_{i}^{-}\right)\left(M_{i}^{+^{\prime}}-M_{i}^{-^{\prime}}\right) .
\end{aligned}
$$

As shown by Eqs. (5) and (6), the model STF depends only on two parameters. One is the Fried parameter, $r_{0}$, which appears in the well-known phase structure function $\mathscr{D}(s)$ and $\left\langle a_{i}^{2}\right\rangle$ (e.g., Noll 1976), and the other is the AO correction efficiency array $\boldsymbol{\beta}$ for the $N$ (partially) corrected KL modes. The four-dimensional integral in Eq. (5) is evaluated using Monte Carlo integration; its convergence has been analyzed in Wöger et al. (2008). The values of $r_{0}$ and $\beta$ are taken as the sole input parameters to the STF model. The sensitivity of the STF model and the resulting photometric precision to these input parameters are discussed in the following sections.

\section{Method}

\subsection{Estimation of input parameters}

The analytical STF model from the previous section takes two input parameters, $r_{0}$ and the array $\boldsymbol{\beta}$. For solar observations, $r_{0}$ has typically been estimated using the spectral ratio method (von der Lühe 1984):

$\epsilon(s)=\frac{|\langle I(s)\rangle|^{2}}{\left\langle|I(s)|^{2}\right\rangle}=\frac{|\langle O(s) \cdot S(s)\rangle|^{2}}{\left\langle|O(s) \cdot S(s)|^{2}\right\rangle}=\frac{|\langle S(s)\rangle|^{2}}{\left\langle|S(s)|^{2}\right\rangle}=\frac{\mathrm{LETF}^{2}}{\mathrm{STF}}$,

where LETF is the long exposure transfer function. In the absence of AO correction, $r_{0}$ is found by fitting a model to the azimuthal average of $|\langle I(s)\rangle|^{2} /\left\langle|I(s)|^{2}\right\rangle$. In the presence of AO correction, estimating $r_{0}$ becomes more complicated because it is difficult to differentiate between wavefront improvements from the AO correction and increased $r_{0}$; this has motivated the introduction of a "generalized Fried parameter" (Cagigal \& Canales 2000). While in theory the object's Fourier signal in Eq. (7) should cancel, Denker et al. (2007) has shown that $\epsilon(s)$ is not independent of the morphological structure in the object and can 

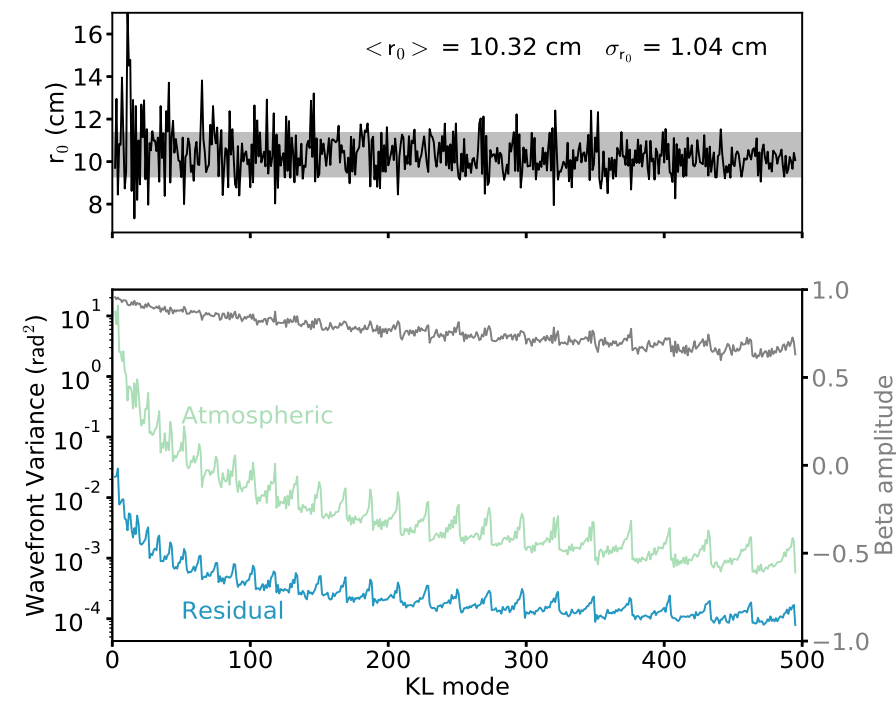
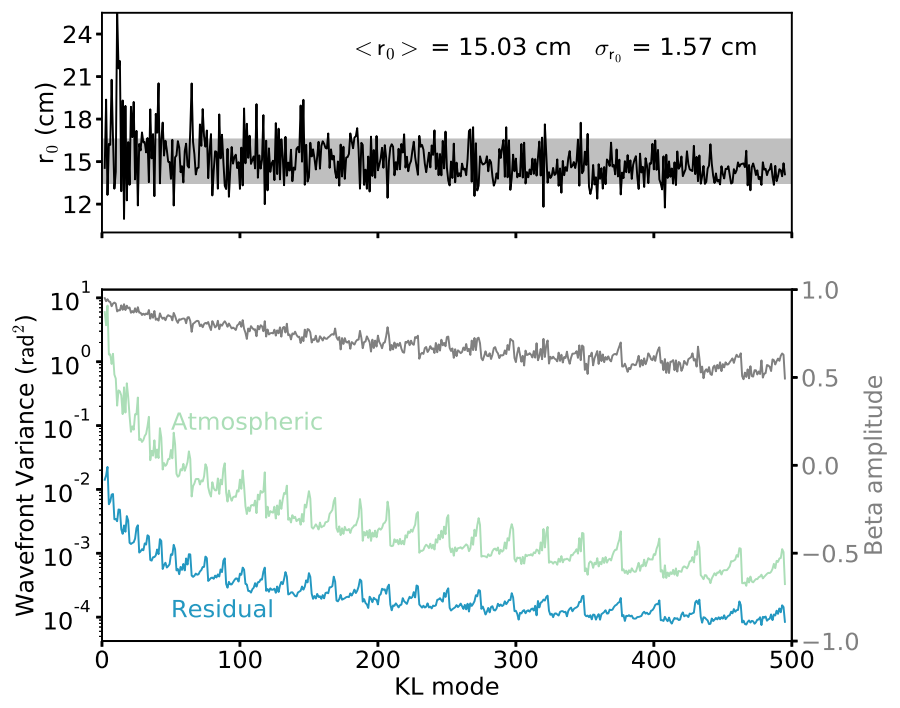

Fig. 1. Top: estimate of $r_{0}$ using the atmospheric variance measured from DM actuator commands for the $10 \mathrm{~cm}(l e f t)$ and $15 \mathrm{~cm}(r i g h t)$ simulated atmospheres. The gray band represents the standard deviation, where both the mean and standard deviation are computed over all KL modes. We note that the two atmospheres are scaled versions of each other. Bottom: Atmospheric (green) and residual (blue) wavefront variances measured from the DM actuators and WFS shifts, respectively. The computed value of $\boldsymbol{\beta}$ from Eq. (8) is shown in gray.

introduce asymmetries in the spectral ratio - and therefore errors in the estimation of $r_{0}$.

We took a different approach to estimate $r_{0}$ and $\beta$ that utilizes AO system measurements of the atmospheric and residual wavefront covariances. Since the covariance of a Kolmogorov power spectrum is well known and scales with $r_{0}$ (Noll 1976), the atmospheric covariance measures $r_{0}$ and the residual covariance measures the AO system performance. The covariances are found respectively as $\mathscr{C}_{\mathrm{atm}}=\mathscr{H}_{\mathrm{DM}} \mathbf{M}_{\mathrm{DM}}\left(\mathscr{H}_{\mathrm{DM}} \mathbf{M}_{\mathrm{DM}}\right)^{\mathrm{T}}$ and $\mathscr{C}_{\text {res }}=\mathscr{H}_{\mathrm{WFS}} \mathbf{M}_{\mathrm{WFS}}\left(\mathscr{H}_{\mathrm{WFS}} \mathbf{M}_{\mathrm{WFS}}\right)^{\mathrm{T}}$. The matrices $\mathscr{H}_{\{\mathrm{DM}, \mathrm{WFS}\}}$ convert DM actuator and WFS shift values to KL modal coefficients, respectively, and are determined from the AO-system calibration. The matrices $\mathbf{M}_{\{\mathrm{DM}, \mathrm{WFS}\}}$ contain the set of all DM actuator and WFS shift values measured during the image burst acquisition, respectively. We estimated WFS noise as the highfrequency offset in the power spectral density of the WFS shifts averaged over all sub-apertures and subtract it from $\mathbf{M}_{\{\mathrm{WFS}\}}$. Noise in cross-correlating SH WFS shift measurements is caused by several sources. Besides photon and detector read noise in the sub-aperture images themselves, other noise contributors are the contrast (and structure) of the imaged source, severity of the seeing conditions, anisoplanatism in individual sub-aperture images, and the cross-correlation peak finding algorithm. Due to the fact that the KL modes are statistically independent, the atmospheric and residual modal variances, $\sigma_{i \text {,atm }}^{2}$ and $\sigma_{i, \text { res }}^{2}$, are simply the diagonal values of the respective covariance matrices. The atmospheric modal variance of Kolmogorov turbulence can be modeled as a function that depends only on the scaling parameter $\left(D / r_{0}\right)^{5 / 3}$ (Noll 1976), where $\mathrm{D}$ is the telescope aperture diameter. Therefore, the value of $r_{0}$ is estimated by comparing the measured atmospheric variance, $\sigma_{\text {atm }}^{2}$, to the theoretical variance and finding the best-fit scaling factor. This approach removes the dependence of $r_{0}$ on the image scene or AO correction.

The AO performance array, $\boldsymbol{\beta}$, is also estimated from $\sigma_{\text {atm }}^{2}$ and $\sigma_{\text {res }}^{2}$. From Wöger $\&$ von der Lühe (2007), $\beta_{i}$ is computed as

$\beta_{i}=1-\sqrt{\frac{\sigma_{i, \mathrm{res}}^{2}}{\sigma_{i, \mathrm{~atm}}^{2}}}$.
The top panels of Fig. 1 shows the $r_{0}$ estimate for the $10 \mathrm{~cm}$ (left) and $15 \mathrm{~cm}$ (right) atmospheres using $\sigma_{\text {atm }}^{2}$ derived from the DM actuator commands and the theoretical KL values for atmospheric turbulence. The mean and standard deviation are taken over the first $500 \mathrm{KL}$ modes. The larger standard deviation in low order modes is due to the fact that these modes evolve on longer timescales than high order modes. This results in fewer sampling statistics of these low order modes, and therefore a larger standard error of the sample mean over the $5 \mathrm{~s}$ time series. The bottom of Fig. 1 shows the values of $\sigma_{\text {atm }}^{2}$ and $\sigma_{\text {res }}^{2}$ from the DM actuator commands and WFS shifts, respectively, as well as the corresponding value of $\boldsymbol{\beta}$ computed using Eq. (8).

\subsection{Estimation of photometric precision}

To evaluate photometric precision, we use high spatial resolution images from numerical simulations consistent with four-meter solar telescope observations. The images are computed with the MURaM code with $16 \mathrm{~km}$ resolution (e.g., Rempel 2014) synthesized at $500 \mathrm{~nm}$ using a radiative transfer code (Uitenbroek 2001). We used one simulation of network and one of internetwork fields so that the simulations have differing intensity amplitude spectra. We convolved the image with the simulated STF of the atmosphere-telescope system, $\mathrm{STF}_{\text {sim }}$, computed directly from the residual phase screens using Eq. (3). We then deconvolved with a model STF computed with a set of input parameters to obtain the reconstructed image. Both $\mathrm{STF}_{\text {sim }}$ and the model STF include the telescope aperture modulation transfer function that cancels out in the convolution and deconvolution process.

We directly compared the original image with the reconstructed image and calculate the photometric precision as the standard deviation of the normalized difference image (original minus reconstructed divided by the original image for all pixels). We repeated this for both image scenes. A burst image, original image, and reconstructed image for each image scene is shown in Fig. 2 using the $10 \mathrm{~cm}$ atmospheric simulations and values of $r_{0}$ and $\beta$ from the left of Fig. 1. The improvement in the Fourier amplitudes by using the reconstruction can be seen by comparing 

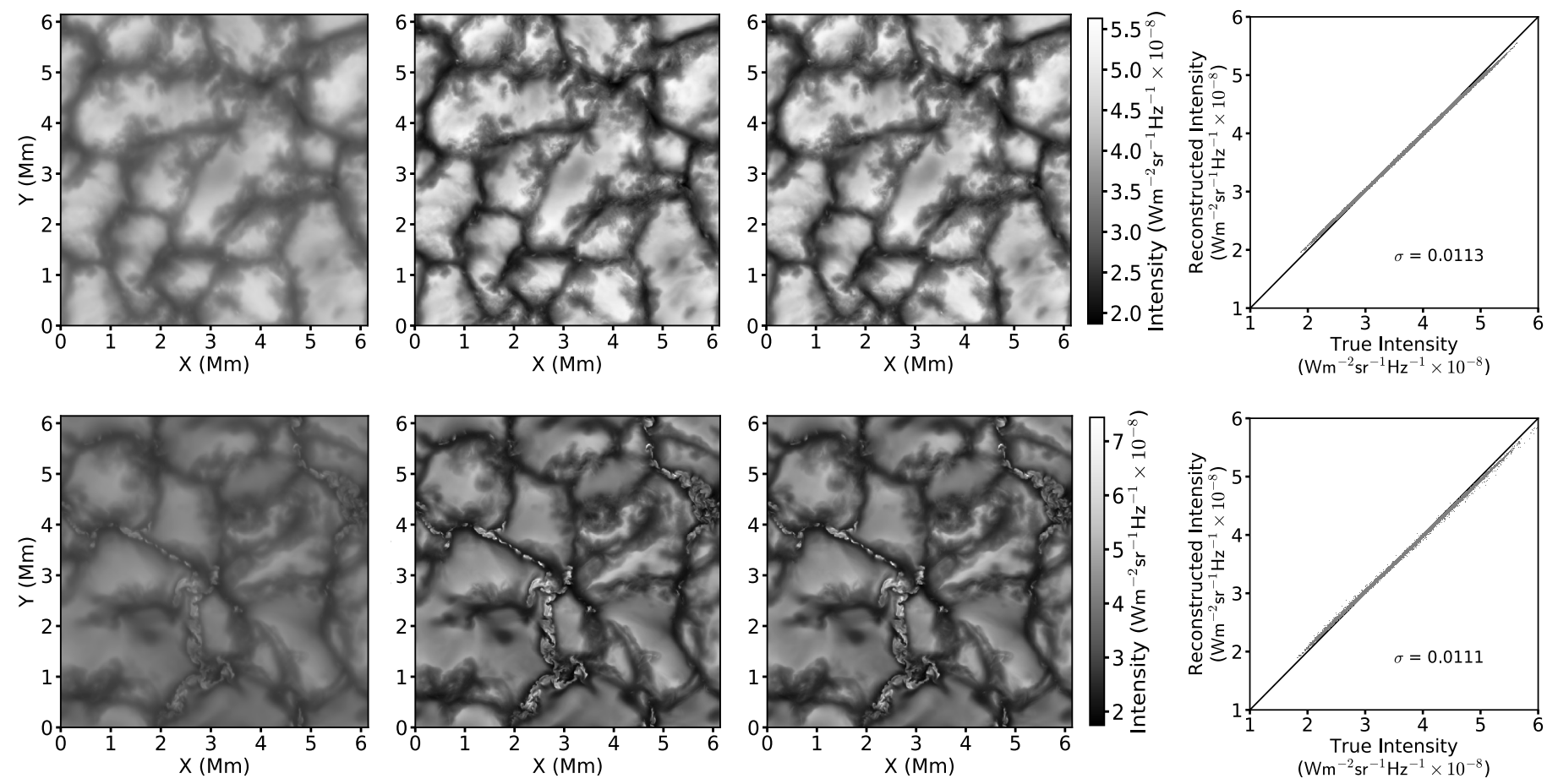

Fig. 2. From left to right: post-AO burst image, original image, reconstructed image, and reconstructed vs. original image precision for the $10 \mathrm{~cm}$ atmosphere. The images were reconstructed with the STF using $r_{0}$ and $\beta$ from Fig. 1. Top: internetwork image. Bottom: network image.

the burst image with the reconstructed image. We note how the burst image appears to resolve small-scale structures, but does not accurately measure the image Fourier amplitudes due to the turbulent atmosphere. The photometric precision is shown on the right of Fig. 2 and is the standard deviation of the intensity difference from the black line.

\section{Results}

In this section, we separately analyze the impact of errors introduced through the parameters $r_{0}$ and $\beta$ on photometric precision. Using the AO-derived values of $r_{0}$ and $\boldsymbol{\beta}$ shown in Fig. 1, we computed the model STFs under three different conditions; varying the Fried parameter input $r_{0}$, varying the number of $\mathrm{KL}$ modes included in $\beta$, and fluctuating the KL mode correction by applying a per mode pseudo-random factor to the $\beta_{i}$ values in $\boldsymbol{\beta}$. The results are shown below.

\subsection{Variations of the Fried parameter $r_{0}$}

We tested the sensitivity of the analytical STF model to uncertainties in the estimation of $r_{0}$, which could result from errors in the WFS measurements or divergence of the atmosphere from a Kolmogorov spectrum. We varied the input value of $r_{0}$ within $20 \%$ of the AO-derived values of 10.32 and $15.03 \mathrm{~cm}$. We computed the model STFs and determined the resulting photometric precision, shown in Fig. 3 for the two image scenes. Also shown are the measured pre- and post-AO corrected $\mathrm{STF}_{\text {sim }}$ and the diffraction-limited squared modulation transfer function, MTF.

As shown, the photometric precision remains within $2 \%$ photometric error for values of $r_{0}$ that are within approximately $10 \%$ and $20 \%$ for the 10 and $15 \mathrm{~cm}$ atmosphere, respectively, where $2 \%$ precision is a requirement of the DKIST Visible Broadband Imager (VBI; Wöger et al. 2012). We note a systematic overestimation in the model STFs with respect to $\mathrm{STF}_{\text {sim }}$ at normalized spatial frequencies $\gtrsim 0.4$. This effect likely contributes to the limit of photometric accuracy achieved in this study. Also shown by the vertical dashed line in the bottom of Fig. 3 is the $r_{0}$ parameter determined from the AO system (from Fig. 1). The derived value of $r_{0}$ for the $10 \mathrm{~cm}$ atmosphere is somewhat larger than the value set in the simulation. This effect could be caused by increased noise in the WFS measurement; the DKIST WFS is designed with a subaperture size of $9.3 \mathrm{~cm}$. Therefore, measured values of $r_{0} \lesssim 9.3 \mathrm{~cm}$ could be attenuated by higher order optical aberrations. We note that the photometric precision is more sensitive to under-estimations of $r_{0}$ than to over-estimations since, due to the scaling of $r_{0}$, the STF is less sensitive to larger values of $r_{0}$. This is also responsible for the higher accuracy of the $15 \mathrm{~cm}$ atmosphere for similar relative variations in $r_{0}$.

\subsection{Changes in the number of included $K L$ modes}

We tested the dependence of the reconstruction on the number of AO-compensated KL modes used in the calculation of the model STFs. Using $r_{0}$ fixed to the AO-derived values (10.32 and $15.03 \mathrm{~cm}$ ), we computed the STF for different numbers of modes included in the parameter array $\beta$ computed from Eq. (8) using the variances from Fig. 1. The resulting model STFs and photometric precision are shown in Fig. 4.

As expected, increasing the number of modes included in the model improves the accuracy of the model STF. As the number of corrected modes increases to several hundred, the improvement in the photometric precision reaches the $2 \%$ threshold. We note the increase in error from 400 to $500 \mathrm{KL}$ modes in the $10 \mathrm{~cm}$ case, which indicates that the WFS had difficulty sensing the highest order modes and therefore worsened the reconstruction accuracy. In the $15 \mathrm{~cm}$ case, it is likely that the accuracy limit in this study is influenced by the number of corrected modes in the simulated AO system (500 KL modes). However, improvements in the photometric accuracy are likely 

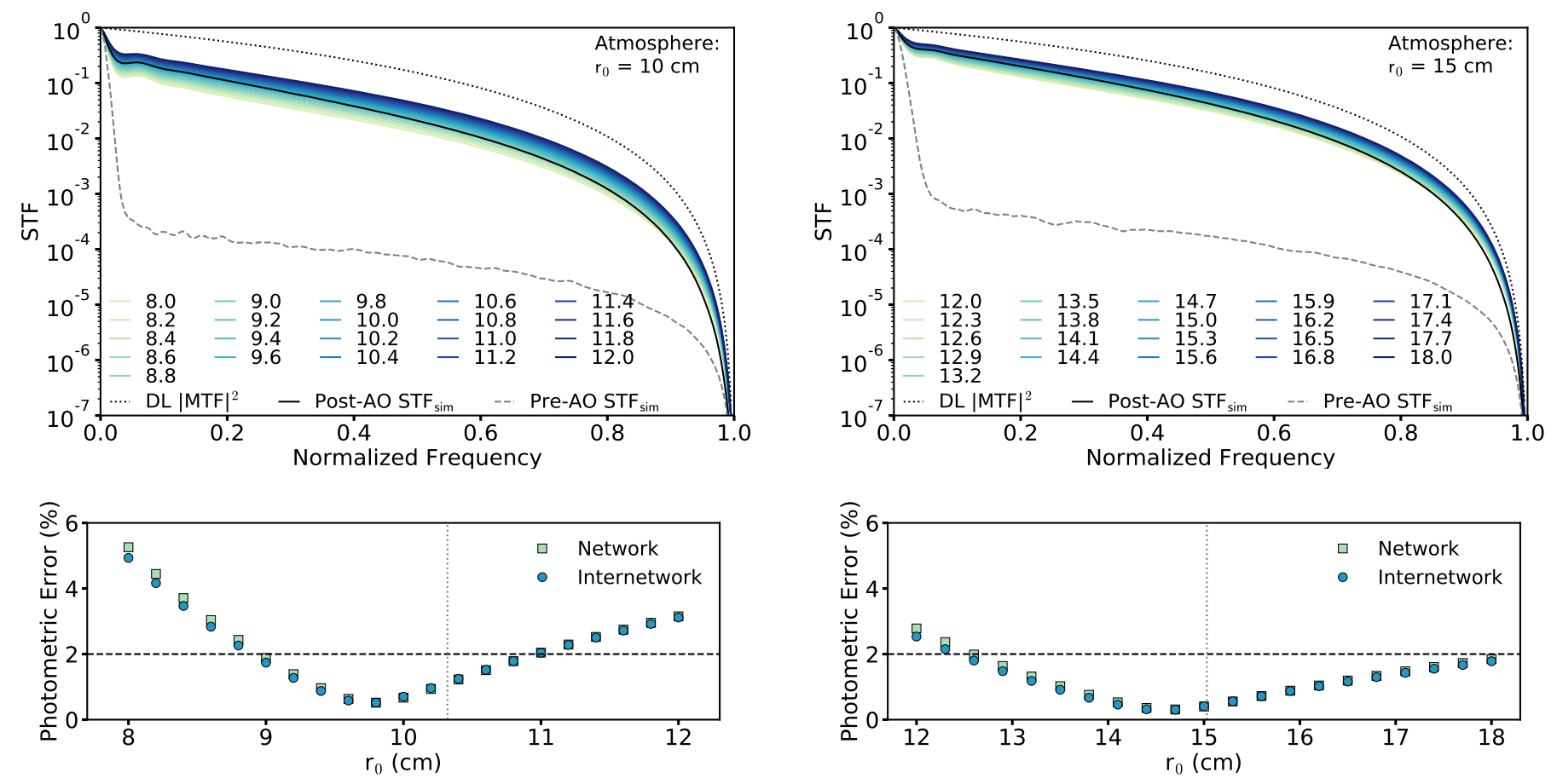

Fig. 3. Top: model STFs computed using $\beta$ from Fig. 1 under different input values of $r_{0}$ in $\mathrm{cm}$ (colored) for the $10 \mathrm{~cm}$ (left) and $15 \mathrm{~cm}($ right)

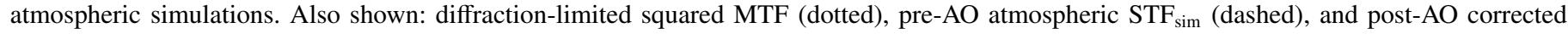
$\mathrm{STF}_{\text {sim }}$ (solid black). Bottom: resulting image photometric error for the two image scenes. The AO-estimated value of $r_{0}$ is shown as the dashed vertical line. The $2 \%$ target value for DKIST is marked with the horizontal line.

to be relatively small and are achieved at the expense of increased computation time.

\subsection{Fluctuations in KL mode corrections}

Uncertainties in the WFS measurements, in addition to affecting the accuracy of the estimated $r_{0}$ values, may also influence the accuracy of the estimated $\beta_{i}$ values. This effect is likely to be larger for higher-order modes, which have more small-scale structure and therefore have lower signal-to-noise ratio in the WFS measurement. To understand the contribution to photometric error, we computed the model STFs using pseudo-random fluctuations of the correction values for the KL modes. The error is modeled as a random error that increases linearly with increasing KL mode, therefore assuming that the WFS has increased difficulty measuring these modes. Each mode $\beta_{i}$ adds an error with a random number $u_{i}$ between -1 and 1 multiplied by a constant such that the highest order mode can not deviate more than $10 \%$ of a fully corrected mode:

$\delta \beta_{i}=\left(\frac{u_{i} \times i}{N}\right) \times 0.1$

where $N=500$ in this study.

We generated 25 pseudo-random fluctuations, which are shown in Fig. 5 overlaid with the original values of $\beta$. We then computed the model STFs using each $\beta$ set and the values of $r_{0}$ fixed to the AO-derived values $(10.32$ and $15.03 \mathrm{~cm})$. The model STFs and corresponding photometric errors are shown in Fig. 5. As shown, the STF changes very little under this random error fluctuation and all STFs are nearly identical. This indicates that random fluctuations in a given KL mode, $\beta_{i}$, have negligible effects on photometric precision.

\section{Discussion}

In this work, we analyzed the influence of the Fourier amplitude recovery algorithm and its inputs - in particular the on-axis STF model - on the photometric precision. In order to isolate the effects of these input parameters, we based the work on several idealized assumptions of the telescope and AO system. A full evaluation of photometric precision in any observed image must be seen in the context of all sources of error that can impact it, including for example other steps in the reconstruction algorithm, source and detector noise, stray light, and off-axis performance of the AO system. These are discussed below and put into the context of the results in this work.

This study focuses on the Fourier amplitude reconstruction which determines the relative intensity weighting of structures at different spatial scales in the image. The photometric properties in the reconstructed image cannot be uncoupled from estimation of the Fourier phases for the reconstruction. In speckle interferometric algorithms, the image Fourier phases are typically estimated using recursive or iterative algorithms, or a combination of both (Matson 1991; de Boer et al. 1992; Wöger et al. 2008). The convergence of the Fourier phase reconstruction algorithms to a unique solution has been explored in the past, for example, in Matson (1991) and Wöger et al. (2008). In general, the unique solution depends primarily on the chosen bispectrum parameters and can thus be influenced by the number of evaluated bispectrum positions and iterations. Pehlemann \& von der Lühe (1989), however, demonstrated that Fourier phases can be recovered with high accuracy as long as a minimum of evaluated bispectrum positions are used. Therefore, in all practical situations, errors in the Fourier amplitude recovery dominate the photometric precision in a reconstructed image over errors in the Fourier phase estimation. 
C. L. Peck et al.: Speckle image reconstruction precision for large solar telescopes
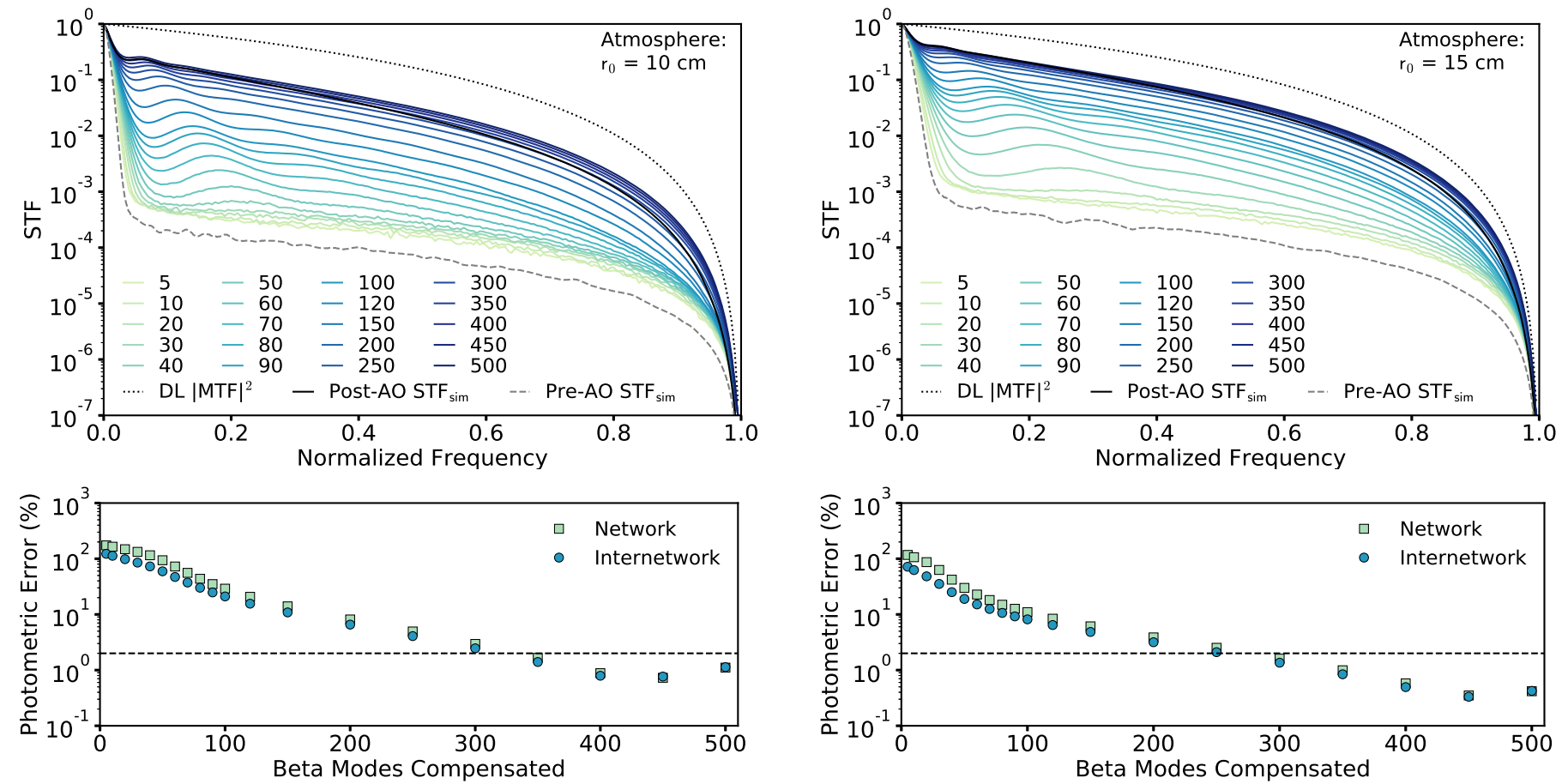

Fig. 4. Top: model STFs for $r_{0}$ and $\boldsymbol{\beta}$ from Fig. 1 where $\boldsymbol{\beta}$ is truncated to the specified number of KL modes (colored). Bottom: resulting image photometric error for the two image scenes. The target $2 \%$ value is marked with the dashed horizontal line.
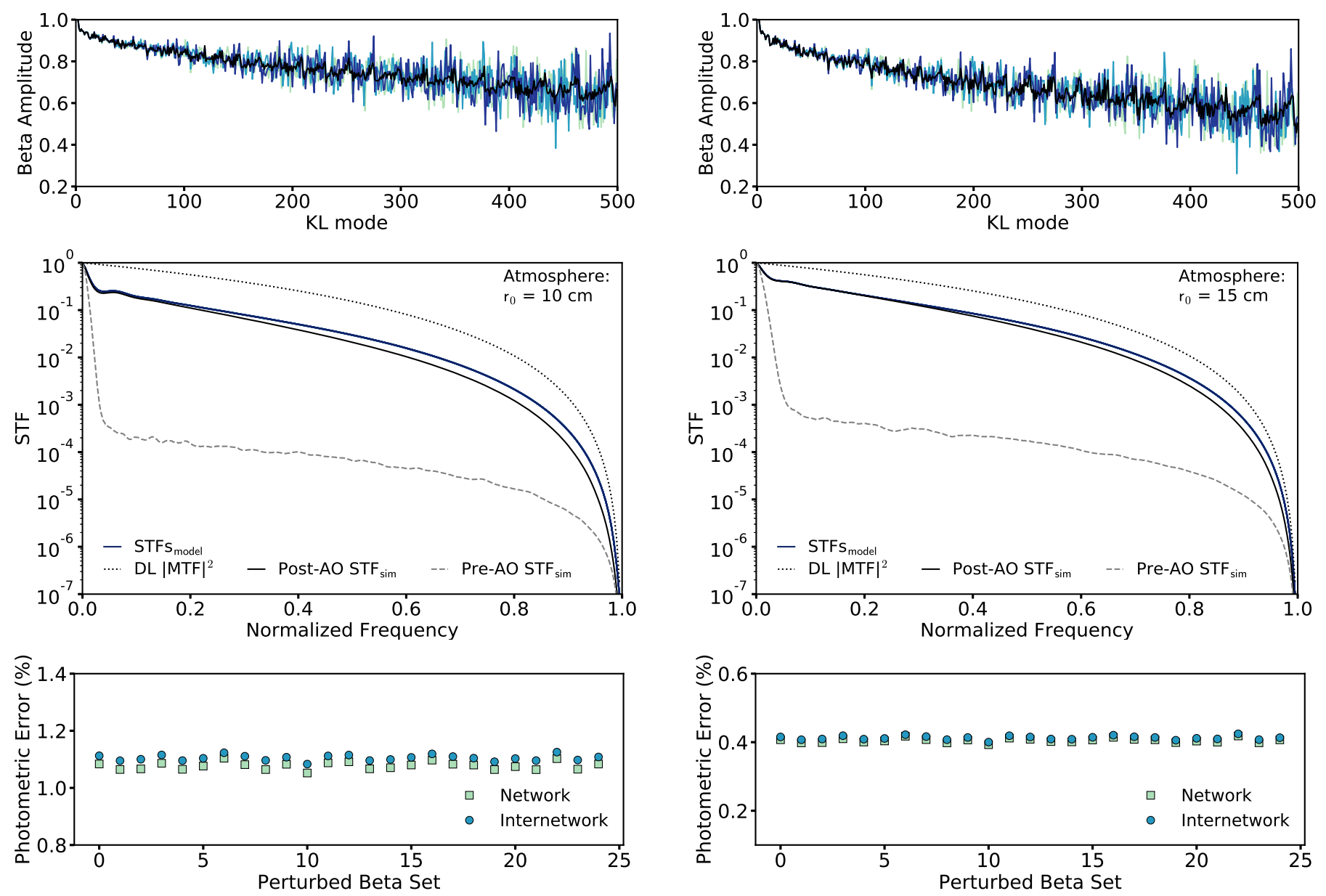

Fig. 5. Top: values of three sets of $\boldsymbol{\beta}$ with pseudo-random fluctuations added on each mode from Eq. (9) and the original $\boldsymbol{\beta}$. Middle: model STFs for $r_{0}$ from Fig. 1 and the perturbed $\beta$ sets (colored). Bottom: resulting image photometric error for the two image scenes. 
Measurements of the solar atmosphere are impacted by several sources of noise, which were deliberately not considered in this study beyond their potential to introduce error in the determination of the model STF input parameters. Detector noise in solar observations is dominated by photon noise, particularly when considering modern scientific CMOS sensors that can achieve less than two photo-electrons of read noise and are consequently more and more often used at telescope facilities such as DKIST. Additionally, detector noise can show characteristics that are particularly inconvenient for extended source imaging and reconstruction algorithms, for example fixed pattern noise which exhibits a non-white signature in the two-dimensional power spectrum of an image. In general, the treatment of noise through filtering in the reconstruction process is a very important aspect that needs careful consideration. In most image reconstruction pipelines, noise is treated by applying a filter function that proportionally dampens amplitudes at those frequencies that have been identified to be affected by noise. Therefore, this process can significantly impact the photometric precision in the final image: for example, if noise in the input images is underestimated the contrast in the reconstruction will be overestimated, while in the opposite case it will be underestimated. Many noise filtering techniques, with a large range of complexity, have been proposed in the past. Some of these have been specifically suggested for solar image processing (e.g., de Boer 1996; DeForest 2017). An evaluation of the large variety of these techniques is outside the scope of this work. Nonetheless, we estimate that improper noise filtering, in particular due to a poor noise estimate affecting some or all spatial frequencies, can easily bias the photometric properties in reconstructed images by several percent.

A final and significant uncertainty that can affect the photometric precision in any image observed with an optical system is stray light. Identifying sources of stray light is often not trivial and measuring stray light can be difficult even with space-borne solar telescopes. The best option is often to measure stray light directly during a transit of a planet or occultation by the moon, and even in these cases a complete characterization is not simple (Wedemeyer-Böhm 2008). Past works (e.g., Danilovic et al. 2008; Wedemeyer-Böhm \& Rouppe van der Voort 2009) have shown for example that for the Hinode space telescope the stray light contribution to granular contrast is likely of the order of a few percent, a reasonable number given its many potential sources.

The three major sources of photometric error discussed here - the applied STF, the noise filtering process, and stray light need to be bounded to allow, for example, comparisons between observed and reconstructed images with radiative magnetohydrodynamical simulations of the solar atmosphere. The results of this work demonstrate that the photometric errors introduced by the on-axis STF can be minimized to well below $1 \%$, and therefore is not fundamentally a limitation to such a comparison. The additional sources of error are independent of a reconstruction algorithm and have to be carefully assessed and considered in data acquired by any telescope.

While this work focused on on-axis STFs, the fielddependence of conventional AO correction has been modeled in the past (Wöger \& von der Lühe 2007). Only turbulent layers located high above the telescope pupil contribute to field dependent variations of the point spread function. A full or partial correction of wavefront distortions conjugated to the telescope pupil - as performed by conventional AO systems - does not affect the spatial decorrelation of modal Zernike coefficients describing these distortions (Molodij \& Rousset 1997) across the field, as described by Wöger \& von der Lühe (2007). Future MCAO systems (e.g., von der Lühe et al. 2005; Berkefeld et al. 2006; Schmidt et al. 2017) aim to correct for discrete turbulent layers in earth's atmosphere using multiple correctors optically conjugated to the respective layer heights. Adapting this work to incorporate the correction by an MCAO system in the STF calculations requires knowledge of the remaining turbulence in the corrected and uncorrected layers so that the spatial decorrelation coefficients of the optical modes can be derived. Furthermore, to apply this particular work to off-axis STFs, whether a conventional $\mathrm{AO}$ or an MCAO system is assumed for correction, an analytical or numerical decorrelation model applicable to KL modes is needed. While this is beyond the scope of this study, it is highly desirable and will be a subject of the authors' future work.

The model STFs for this work were computed for an unobstructed circular telescope aperture such as that of the Goode Solar Telescope (Goode \& Cao 2012), or DKIST. This was convenient for several reasons, in particular because it allowed easy and direct cross-verification of all results generated by the AO simulation tool and the computer code that calculated the analytical STF models. The model STFs can easily be computed for telescopes with a central obscuration incurred by onaxis Gregorian style telescopes, such as the GREGOR telescope (Schmidt et al. 2012), or EST. In fact, the implementation of KL modal functions for computation of model STFs in this work is based on Cannon (1996) which can accommodate a circular obstruction of any size with a single parameter, and can simultaneously calculate both the modal coefficient variances and modal functions for these cases. Furthermore, this algorithm can incorporate any atmospheric structure function if so desired, although we strictly used the original Kolmogorov structure function which has in practice been verified as being a good assumption for most telescope locations. In addition to errors introduced by the input parameters studied in this work, the sensitivity of the photometric precision to the central obscuration parameter should be analyzed for model STFs applicable for telescopes like GREGOR or EST.

Finally, results from this work are relevant to investigations of photometric precision in images reconstructed using algorithms other than speckle including Multi-Object Multi-Frame Blind Deconvolution (van Noort et al. 2005) and Joint Phase Diverse Speckle (Paxman et al. 1992). Some research has been performed to analyze potential ways to improve on the photometric performance of reconstructed images using these algorithms (Scharmer et al. 2010). The authors of that work suggest to incorporate into the original reconstruction algorithms the application of a transfer function that is based on two components: a) an estimate of the low-order modal aberrations derived from the phase estimation algorithms themselves; and b) a statistical estimate of the (uncorrected) high-order modal aberrations derived from the prevailing seeing conditions. The latter requires an accurate estimation of $r_{0}$, but it remains unclear to what accuracy the value must be known to achieve a particular precision. We surmise that had the authors performed a similar sensitivity investigation of photometric precision versus $r_{0}$ value, they would have reached a similar conclussion.

\section{Conclusion}

In this work, we present the results of our analysis of the sensitivity of analytical models for the on-axis STF to their input parameters. These are the Fried parameter $r_{0}$ and the AO system performance as described by an efficiency array, $\boldsymbol{\beta}$. We estimated 
these parameters from the AO system control data. This study separates various mechanisms that can affect the model STF accuracy by comparing the model STFs with the post-AO STF of the atmospheric and AO system simulations. We analyzed the sensitivity of the model STF on the photometric properties of high spatial resolution images of the solar photosphere as they would be acquired with a $4 \mathrm{~m}$ aperture telescope such as DKIST.

We examined the sensitivity of the model STF to inaccuracies in determining $r_{0}$, and its impact on the photometric precision in images calibrated with this STF. Using the AO-derived values of $\boldsymbol{\beta}$ from Fig. 1, we varied $r_{0}$ up to $20 \%$ from the nominal values and found that deviations in $r_{0}$ greater than $10 \%$ and $20 \%$ in the 10 and $15 \mathrm{~cm}$ atmosphere, respectively, degraded the photometric precision to more than $2 \%$. The ability to correctly determine $r_{0}$ is the most important factor in photometric precision as this parameter appears to have the largest impact on the shape of the STF. This result could prove to be useful for enhancing the expected photometric precision in images deconvolved with long-exposure point-spread functions derived from AO control data (e.g., Veran et al. 1997; Marino \& Rimmele 2010).

We evaluated the effect of the number of KL modes included in the analytical model of the STF on the photometric precision. We computed the STFs with different numbers of KL modes by modifying the length of the input parameter array $\boldsymbol{\beta}$ and using the values of $r_{0}$ derived from the AO system. We find that several hundred KL modes are required to achieve the minimum precision requirements of the DKIST VBI. The results also indicate that the photometric precision could be improved with the inclusion of more than 500 corrected modes, but the improvements would be limited and at the expense of longer computation times.

Finally, to determine the effect of uncertainty in the measurement of the AO efficiency, we added pseudo-random noise to each entry in the original $\boldsymbol{\beta}$ array. We find that these fluctuations have negligible effect on the photometric precision and deduce that the shape of the $\beta$ profile plays a larger role than the relative amplitudes of neighboring modes.

This study provides the first rigorous analysis of the process of utilizing AO system information to derive both an estimate for the Fried parameter, $r_{0}$, and $\beta$ in order to support the speckle image reconstruction process. The main advantage of this method is that it removes the dependence of the $r_{0}$ estimate on the image scene (via non-azimuthally symmetric power spectra). For the nominal values of these parameters, we find a photometric error below $1 \%$ and with little dependence on image scene. Furthermore, this work provides for the first time direct evidence that the parameter $\boldsymbol{\beta}$ accurately encodes the impact of an AO system on the model STF, and that the analytical model described in Eq. (5) can indeed be used to calibrate the Fourier amplitudes during the image reconstruction process. While previous works had already indicated high accuracy of the applied calibration function (e.g., Wöger et al. 2008), this study is able to address several aspects of its sensitivity in more detail.

Acknowledgements. The authors would like to thank the anonymous referee whose comments contributed to significant improvements to the manuscript. The National Solar Observatory is operated by the Association of Universities for Research in Astronomy (AURA) Inc. under a cooperative agreement with the National Science Foundation. This material is based upon work supported by the National Science Foundation Graduate Research Fellowship Program under grant No. DGE 1144083 and NASA award number NNX12AB35G. This work utilized the Janus supercomputer, which is supported by the National Science
Foundation (award number CNS-0821794), the University of Colorado Boulder, the University of Colorado Denver, and the National Center for Atmospheric Research. Janus is operated by the University of Colorado Boulder. C.L.P. would like to thank Dr. Mark Rast for valuable discussions during the course of this research.

\section{References}

Berkefeld, T., Soltau, D., \& von der Lühe, O. 2006, in Proc. SPIE, 6272, 627205 Cagigal, M. P., \& Canales, V. F. 2000, Opt. Soc. Am. A, 17, 903

Cannon, R. C. 1996, Opt. Soc. Am. A, 13, 862

Collados, M., Bettonvil, F., Cavaller, L., et al. 2013, Mem. Soc. Astron. Ital., 84, 379

Dai, G.-M. 1995, Opt. Soc. Am. A, 12, 2182

Danilovic, S., Gandorfer, A., Lagg, A., et al. 2008, A\&A, 484, L17

de Boer, C. R. 1996, A\&AS, 120, 195

de Boer, C. R., Kneer, F., \& Nesis, A. 1992, A\&A, 257, L4

DeForest, C. E. 2017, ApJ, 838, 155

Denker, C., Deng, N., Rimmele, T. R., Tritschler, A., \& Verdoni, A. 2007, Sol. Phys., 241, 411

Elmore, D. F., Rimmele, T., Casini, R., et al. 2014, in Ground-based and Airborne Instrumentation for Astronomy V, Proc. SPIE, 9147, 914707

Fried, D. L. 1966, J. Opt. Soc. Am., 56, 1380

Gavel, D. T. 2003, in Adaptive Optical System Technologies II, eds. P. L. Wizinowich, \& D. Bonaccini, Proc. SPIE, 4839, 972

Goode, P. R., \& Cao, W. 2012, in Second ATST-EAST Meeting: Magnetic Fields from the Photosphere to the Corona., eds. T. R. Rimmele, A. Tritschler, F. Wöger, et al., ASP Conf. Ser., 463, 357

Johansson, E. M., \& Gavel, D. T. 1994, in Amplitude and Intensity Spatial Interferometry II, ed. J. B. Breckinridge, Proc. SPIE, 2200, 372

Johnson, L. C., Cummings, K., Drobilek, M., et al. 2014, in Adaptive Optics Systems IV, Proc. SPIE, 9148, 91481

Knox, K. T., \& Thompson, B. J. 1974, ApJ, 193, L45

Korff, D. 1973, Opt. Soc. Am., 63, 971

Labeyrie, A. 1970, A\&A, 6, 85

Lohmann, A. W., Weigelt, G., \& Wirnitzer, B. 1983, Appl. Opt., 22, 4028

Marino, J. 2012, Opt. Eng., 51, 101709

Marino, J., \& Rimmele, T. 2010, Appl. Opt., 49, G95

Matson, C. L. 1991, Opt. Soc. Am. A, 8, 1905

Matthews, S. A., Collados, M., Mathioudakis, M., \& Erdelyi, R. 2016, in Ground-based and Airborne Instrumentation for Astronomy VI, Proc. SPIE, 9908, 990809

Mikurda, K., \& von der Lühe, O. 2006, Sol. Phys., 235, 31

Molodij, G., \& Rousset, G. 1997, Opt. Soc. Am. A, 14, 1949

Noll, R. J. 1976, Opt. Soc. Am., 66, 207

Paxman, R. G., Schulz, T. J., \& Fienup, J. R. 1992, Opt. Soc. Am. A, 9, 1072

Pehlemann, E., \& von der Lühe, O. 1989, A\&A, 216, 337

Puschmann, K. G., \& Sailer, M. 2006, A\&A, 454, 1011

Rempel, M. 2014, ApJ, 789, 132

Scharmer, G. B., Löfdahl, M. G., van Werkhoven, T. I. M., \& de la Cruz Rodríguez, J. 2010, A\&A, 521, A68

Schmidt, W., von der Lühe, O., Volkmer, R., et al. 2012, Astron. Nachr., 333, 796

Schmidt, D., Gorceix, N., Goode, P. R., et al. 2017, A\&A, 597, L8

Tritschler, A., Rimmele, T. R., Berukoff, S., et al. 2016, Astron. Nachr., 337, 1064

Uitenbroek, H. 2001, ApJ, 557, 389

van Noort, M., Rouppe van der Voort, L., \& Löfdahl, M. G. 2005, Sol. Phys., 228, 191

Veran, J.-P., Rigaut, F., Maitre, H., \& Rouan, D. 1997, Opt. Soc. Am. A, 14, 3057 von der Lühe, O. 1984, Opt. Soc. Am. A, 1, 510

von der Lühe, O., Berkefeld, T., \& Soltau, D. 2005, Comptes Rendus Physique, 6,1139

Wang, J. Y., \& Markey, J. K. 1978, Opt. Soc. Am., 68, 78

Wedemeyer-Böhm, S. 2008, A\&A, 487, 399

Wedemeyer-Böhm, S., \& Rouppe van der Voort, L. 2009, A\&A, 503, 225

Wöger, F., \& von der Lühe, O. 2007, Appl. Opt., 46, 8015

Wöger, F., von der Lühe, O., \& Reardon, K. 2008, A\&A, 488, 375

Wöger, F., McBride, W., Ferayorni, A., et al. 2012, in Second ATST-EAST

Meeting: Magnetic Fields from the Photosphere to the Corona., eds. T. R.

Rimmele, A. Tritschler, F. Wöger, et al., ASP Conf. Ser., 463, 431 